\title{
Assessment of conservative load transfer for fluid-solid interface with non-matching meshes
}

\author{
R. K. Jaiman ${ }^{1}$, X. Jiao ${ }^{2}$, P. H. Geubelle ${ }^{1,2, *, \dagger}$ and E. Loth ${ }^{1}$ \\ ${ }^{1}$ Department of Aerospace Engineering, University of Illinois, Urbana, IL 61801, U.S.A. \\ ${ }^{2}$ Computational Science and Engineering, University of Illinois, Urbana, IL 61801, U.S.A.
}

\begin{abstract}
SUMMARY
We present a detailed comparative study of three conservative schemes used to transfer interface loads in fluid-solid interaction simulations involving non-matching meshes. The three load transfer schemes investigated are the node-projection scheme, the quadrature-projection scheme and the common-refinement based scheme. The accuracy associated with these schemes is assessed with the aid of 2-D fluid-solid interaction problems of increasing complexity. This includes a static load transfer and three transient problems, namely, elastic piston, superseismic shock and flexible inhibitor involving large deformations. We show how the load transfer schemes may affect the accuracy of the solutions along the fluid-solid interface and in the fluid and solid domains. We introduce a grid mismatching function which correlates well with the errors of the traditional load transfer schemes. We also compare the computational costs of these load transfer schemes. Copyright (c) 2005 John Wiley \& Sons, Ltd.
\end{abstract}

KEY WORDS: fluid-solid interaction; load transfer; non-matching meshes; node-projection; quadratureprojection; common-refinement; superseismic; inhibitor; large deformation; grid mismatching function

\section{INTRODUCTION}

Computational methods for fluid-solid interaction (FSI) simulations can be classified as tightlyor loosely-coupled. The tightly-coupled (monolithic) approach solves the fluid and solid equations in a single computational domain using a single numerical framework [1]. The unified treatment of both media tend to improve stability of this method. However, this coupling approach often suffers from the ill-conditioning of the associated matrices and from the inability

${ }^{*}$ Correspondence to: P. H. Geubelle, Department of Aerospace Engineering, University of Illinois, 306 Talbot Lab, 104 S. Wright St., Urbana, IL 61801, U.S.A.

†E-mail: geubelle@uiuc.edu

Contract/grant sponsor: Center for Simulation of Advanced Rockets (CSAR); contract/grant number: B523819 Contract/grant sponsor: University of Illinois 
to use existing fluid and solid solvers. These two disadvantages are addressed with the looselycoupled (partitioned) approach [2], which uses separate and independent techniques for the fluid and solid subdomains and exchanges data along the fluid-solid interfaces (i.e. transfer of jump conditions for kinematic compatibility and dynamic equilibrium) via a fixed-point iteration scheme. The loosely-coupled approach can thus take full advantage of existing, well developed and tested codes for fluid and solid analysis. Solvers with disparate discretization scheme can be 'plugged' into a loosely-coupled framework, with the only changes of obtaining the appropriate boundary conditions from the framework.

The loose coupling approach presents two key areas of challenges. The first one is concerned with the stability, accuracy and convergence of staggered time-integration schemes used for the partitioned approach $[2,3]$. The second one, which is the primary focus of this paper, deals with data transfer between differing mesh representations of the fluid and the solid subdomains. Such meshes are in general non-matching (i.e. have disparate nodal connectivities and positions along the interface) because of differing mesh resolution requirements or discretization schemes. For example, the fluid system usually requires a finer mesh to capture non-linear features such as shock waves and boundary layers. In addition, different types of elements used by the solvers and discretization errors in the respective subdomain geometries can also cause mesh mismatch on the interface.

In this paper, we consider the problem of enforcing the equilibrium on the fluid-solid interface boundary by transferring the fluid traction across a discrete mesh interface, and applying the traction boundary condition on the 'wet' surface of the solid. This load transfer between nonmatching meshes must be numerically accurate (small errors in a specific norm) and physically conservative (balance of loads). While several schemes can ensure conservation [4-6], their levels of the accuracy in load transfer differ substantially and have significant impact on the solutions of the coupled system.

Our emphasis hereafter is to study the accuracy of conservative load transfer in the coupledfield problems with an aid of explicit 2-D loosely coupled FSI framework using a partitioned approach. The framework combines a compressible fluid solver, an explicit elastodynamic solid solver, and three conservative load-transfer schemes: the node-projection scheme of Farhat et al. [4], the quadrature-projection scheme of Cebral and Löhner [5], and the commonrefinement based scheme of Jiao and Heath [6]. Although the comparative examples presented hereafter are 2-D, all these load transfer schemes have been used in 3-D FSI simulations. A short discussion of the computational costs and 3-D extension of these schemes are provided at the end of this paper.

The detailed and systematic comparative study of the accuracy of these three load-transfer schemes is presented using a 1-D static analysis and three transient fluid-solid interaction problems. The first two FSI problems have analytical solutions and allow us to determine the predictive accuracy of the coupling. The first transient problem is 1-D in nature and consists of expansion fans in the fluid and a compression wave in the solid. The next transient problem involves a superseismic shock load over an isotropic elastic solid. Both problems have fully coupled fluid-solid interactions and are relevant to the problems of high pressure/speed compressible flow interacting with solid materials (e.g. blast waves, pressurized piston). Finally, to assess the precision of the load transfer schemes in problems involving large dynamic deflections, we simulate a flexible inhibitor protruding into the core flow.

The outline of this paper is as follows. Section 2 describes the interface coupling conditions and the load transfer schemes. Sections 3-6 present the spatial accuracy comparison of the load 
transfer schemes for the FSI problems of increasing complexity. Section 7 provides a short discussion about cost evaluation and 3-D aspects of the load-transfer.

\section{GENERALIZED INTERFACE COMPUTATIONS FOR PARTITIONED APPROACH}

In the partitioned approach, the decomposed subdomains of the fluid and solid share a common interface boundary. In computational aeroelastic applications, the solid equations are conventionally formulated in Lagrangian co-ordinates on a mesh that moves along with the material, while the fluid equations are formulated in Eulerian co-ordinates, where the mesh serves as a fixed reference for the fluid motion. In a partitioned approach for FSI, the fluid and solid components are solved on separate computational domains, and the aforementioned formulations require moving the fluid mesh at each time step. To avoid severe grid distortions under large deformations, this paper adopts the three-field arbitrary Lagrangian-Eulerian (ALE) formulation proposed in Reference [7]. In this formulation, the moving fluid mesh is treated a third field solved as a pseudo-elastodynamic system.

As illustrated in Figure 1, the coupling of the subdomains is done through exchanging boundary conditions along the interface by imposing compatibility and equilibrium conditions. This section describes the numerical schemes for transferring boundary conditions across the discrete fluid-solid interface. Although non-conservative interpolation-based schemes have been proposed for FSI applications [8], we focus on conservative transfer schemes as conservation has been found to be crucial for the repeated data transfer of large spatial gradients (e.g. shocks) $[4,5]$ and for the stability of partitioned based method [9].

\subsection{Interface conditions and requirements}

At each FSI cycle, two interface boundary conditions corresponding to continuity of tractions and velocities, must be satisfied along the fluid-solid interface $\Gamma_{\mathrm{f} / \mathrm{s}}$, or simply $\Gamma$. Let $\mathbf{t}_{\mathrm{f}}$ and

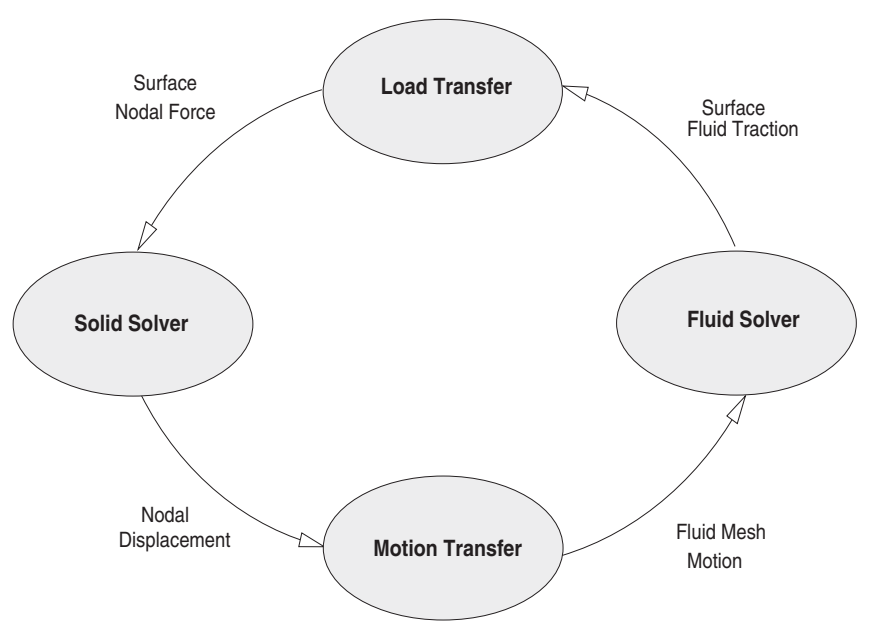

Figure 1. FSI simulation cycle for discrete interface. 
$\mathbf{u}_{\mathrm{f}}$ denote the fluid traction vector and the fluid displacement fields along $\Gamma_{\mathrm{f}}$, respectively, and $\mathbf{t}_{\mathrm{s}}$ and $\mathbf{u}_{\mathrm{s}}$ denote the solid traction vector and the solid displacement fields along $\Gamma_{\mathrm{s}}$. The equilibrium of tractions and compatibility of velocity field can be expressed as

$$
\begin{aligned}
\mathbf{t}_{\mathrm{s}} & =\mathbf{t}_{\mathrm{f}} \\
\frac{\partial \mathbf{u}_{\mathrm{s}}}{\partial t} & =\frac{\partial \mathbf{u}_{\mathrm{f}}}{\partial t}
\end{aligned}
$$

where $\mathbf{t}_{\mathrm{f}}=-p_{\mathrm{f}} \mathbf{n}_{\mathrm{f}}+\boldsymbol{\sigma}_{\mathrm{f}} \cdot \mathbf{n}_{\mathrm{f}}$ and $\mathbf{t}_{\mathrm{s}}=\boldsymbol{\sigma}_{\mathrm{s}} \cdot \mathbf{n}_{\mathrm{s}}$. Here, $p_{\mathrm{f}}$ is the fluid pressure along the interface; $\boldsymbol{\sigma}_{\mathrm{f}}$ and $\boldsymbol{\sigma}_{\mathrm{s}}$ are the fluid viscous tensor and solid stress tensor, respectively; $\mathbf{n}_{\mathrm{f}}$ and $\mathbf{n}_{\mathrm{s}}$ are the normals at the fluid interface $\Gamma_{\mathrm{f}}$ and solid interface $\Gamma_{\mathrm{s}}$, respectively. For inviscid flows, Equation (2) is replaced by the slip-wall boundary condition

$$
\frac{\partial \mathbf{u}_{\mathrm{s}}}{\partial t} \cdot \mathbf{n}_{\mathrm{s}}=\frac{\partial \mathbf{u}_{\mathrm{f}}}{\partial t} \cdot \mathbf{n}_{\mathrm{f}}
$$

and the fluid traction vector in Equation (1) only includes the effect of the pressure field, i.e. $\mathbf{t}_{\mathrm{f}}=-p_{\mathrm{f}} \mathbf{n}_{\mathrm{f}}$.

For matching interfaces where $\Gamma_{\mathrm{f}}$ and $\Gamma_{\mathrm{s}}$ have the same geometry, $\mathbf{n}_{\mathrm{f}}$ and $\mathbf{n}_{\mathrm{s}}$ are along the same directions with opposite signs. However, for non-matching interfaces, their geometries and associated normal directions can in general differ due to discretization errors. To avoid ambiguity, a common normal direction $\hat{\mathbf{n}}$ can be defined as a linear combination of $\mathbf{n}_{\mathrm{f}}$ and $\mathbf{n}_{\mathrm{s}}$, i.e. $\hat{\mathbf{n}}_{\mathrm{f}} \equiv \mathbf{f}\left(\mathbf{n}_{\mathrm{f}}, \mathbf{n}_{\mathrm{s}}\right)$ and $\hat{\mathbf{n}}_{\mathrm{s}} \equiv-\hat{\mathbf{n}}_{\mathrm{f}}$.

In general, while the fluid system addresses the traction field vector $\mathbf{t}_{\mathrm{f}}$ on the element surfaces on the interface, the solid system is solved based on a set of concentrated forces at the nodes on the interface. A distributed fluid load, therefore, must be transferred into equivalent nodal forces along the solid interface. Such a transformation must satisfy conservation of loads, i.e. the solid nodal load vector must yield the same net load vector as the original distributed fluid traction loads. Let $m_{\mathrm{f}}$ and $m_{\mathrm{s}}$ be the number of fluid and solid nodes on the fluid and solid interface meshes, respectively. Let $\mathscr{R}_{\mathrm{f}} \equiv \int_{\Gamma} \mathbf{t}_{\mathrm{f}} \mathrm{d} \Gamma$ denote the fluid load vector and $\mathscr{R}_{\mathrm{S}} \equiv \int_{\Gamma} \mathbf{t}_{\mathrm{s}} \mathrm{d} \Gamma$ the solid load vector. Conservation of loads can be expressed as

$$
\mathscr{R}_{\mathrm{S}}=\sum_{j=1}^{m_{\mathrm{S}}} \mathscr{R}_{\mathrm{s}}^{j}=\sum_{i=1}^{m_{\mathrm{f}}} \mathscr{R}_{\mathrm{f}}^{i}=\mathscr{R}_{\mathrm{f}}
$$

where the $\mathscr{R}_{\mathrm{f}}^{i}$ is the concentrated force associated with the fluid node $i$, and $\mathscr{R}_{\mathrm{s}}^{j}$ is associated with the solid node $j$.

However, the conservation of loads can be satisfied by an infinite number of possible nodal load vectors. To be physically consistent and meaningful, the transformation of load must also be highly accurate, i.e. to have a small errors in a specific norm, and, preferably, the conservation of energy should be satisfied. For the evaluation of the energy, the definition of the virtual work can be conveniently used $[4,6]$.

\subsection{Load transfer formulation}

We describe a formulation for conservative load transfer (i.e. for which total load on the solid surface is exactly that on the fluid surface) based on a Galerkin weighted residual method. This formulation is similar to the mortar element method used in domain decomposition [10] 
and in contact mechanics [11]. This scheme also leads to an energy conservation across the fluid-solid interface under certain conditions $[4,6,12]$.

Let $N_{\mathrm{f}}^{i}$ and $N_{\mathrm{s}}^{j}$ denote the standard finite element shape functions associated with node $i$ of the fluid and node $j$ of the solid interface mesh, respectively, and $\tilde{\mathbf{t}}_{\mathrm{f}}^{i}$ and $\tilde{\mathbf{t}}_{\mathrm{s}}^{j}$ the approximate tractions at the corresponding nodes. The traction fields $\mathbf{t}_{\mathrm{f}}$ and $\mathbf{t}_{\mathrm{s}}$ over $\Gamma_{\mathrm{f}}$ and $\Gamma_{\mathrm{s}}$ are interpolated as

$$
\mathbf{t}_{\mathrm{f}}(\mathbf{x}) \approx \sum_{i=1}^{m_{\mathrm{f}}} N_{\mathrm{f}}^{i}(\mathbf{x}) \tilde{\mathbf{t}}_{\mathrm{f}}^{i}, \quad \mathbf{t}_{\mathrm{s}}(\mathbf{x}) \approx \sum_{j=1}^{m_{\mathrm{s}}} N_{\mathrm{s}}^{j}(\mathbf{x}) \tilde{\mathbf{t}}_{\mathrm{s}}^{j}
$$

respectively. The transfer of distributed loads is therefore to solve for $\tilde{\mathbf{t}}_{\mathrm{s}}^{j}$ given $\tilde{\mathbf{t}}_{\mathrm{f}}^{i}, N_{\mathrm{f}}^{i}$, and $N_{\mathrm{s}}^{j}$. To obtain good approximations for $\tilde{\mathbf{t}}_{\mathrm{s}}$, we minimize the $L_{2}$ norm of the residual $\mathbf{t}_{\mathrm{s}}-\mathbf{t}_{\mathrm{f}}$, i.e.

$$
\left\|\mathbf{t}_{\mathrm{s}}-\mathbf{t}_{\mathrm{f}}\right\|_{2}=\sqrt{\int_{\Gamma}\left(\mathbf{t}_{\mathrm{s}}-\mathbf{t}_{\mathrm{f}}\right)^{\mathrm{T}}\left(\mathbf{t}_{\mathrm{s}}-\mathbf{t}_{\mathrm{f}}\right) \mathrm{d} \Gamma}
$$

This minimization problem can be solved with the aid of the Galerkin weighted residual method by multiplying both sides by a set of weighting functions $\left(\mathbf{W}^{i}=N_{\mathrm{s}}^{i}\right)$ and integrating over the interface boundary $\Gamma$, i.e.

$$
\int_{\Gamma} \mathbf{W}^{i} \mathbf{t}_{\mathrm{s}} \mathrm{d} \Gamma=\int_{\Gamma} \mathbf{W}^{i} \mathbf{t}_{\mathrm{f}} \mathrm{d} \Gamma
$$

From the above derivation, we obtain a discrete linear system for load transfer

$$
\left[\mathbf{M}_{\mathrm{s}}\right]\left\{\tilde{\mathbf{t}}_{\mathrm{s}}\right\}=\left\{\mathscr{R}_{\mathrm{s}}\right\}
$$

where $\left[\mathbf{M}_{\mathrm{s}}\right]$ is the consistent mass matrix for solid interface elements and is defined by

$$
\left[\mathbf{M}_{\mathrm{s}}^{i j}\right]=\int_{\Gamma} N_{\mathrm{s}}^{i} N_{\mathrm{s}}^{j} \mathrm{~d} \Gamma
$$

and $\left\{\mathscr{R}_{\mathrm{S}}\right\}$ is the concentrated force vector to be used by the solid solver,

$$
\left\{\mathscr{R}_{\mathrm{s}}^{j}\right\}=\int_{\Gamma} N_{\mathrm{s}}^{i} \tilde{\mathbf{t}}_{\mathrm{f}} \mathrm{d} \Gamma
$$

This semi-discrete form in Equation (7) is conservative from the summation property of the shape functions, $\sum_{j} N_{\mathrm{s}}^{j}=1$. In typical FSI applications, it is sufficient to evaluate Equation (9) without solving Equation (7) for $\tilde{\mathbf{t}}_{\mathrm{s}}$. However, Equation (7) provides a useful tool in the analysis of errors in $\left\{\mathscr{R}_{\mathrm{S}}^{j}\right\}$, and allows to extract the traction vector for the solid solvers which supports only tractions as the boundary conditions.

Similar treatment of the interface displacement condition can be derived using Equation (2). However, one may choose to transfer the displacements by a pointwise interpolation scheme, as conservation is not required for the moving fluid system analysis. The following subsection describes the integration scheme for evaluating the nodal load vectors. 


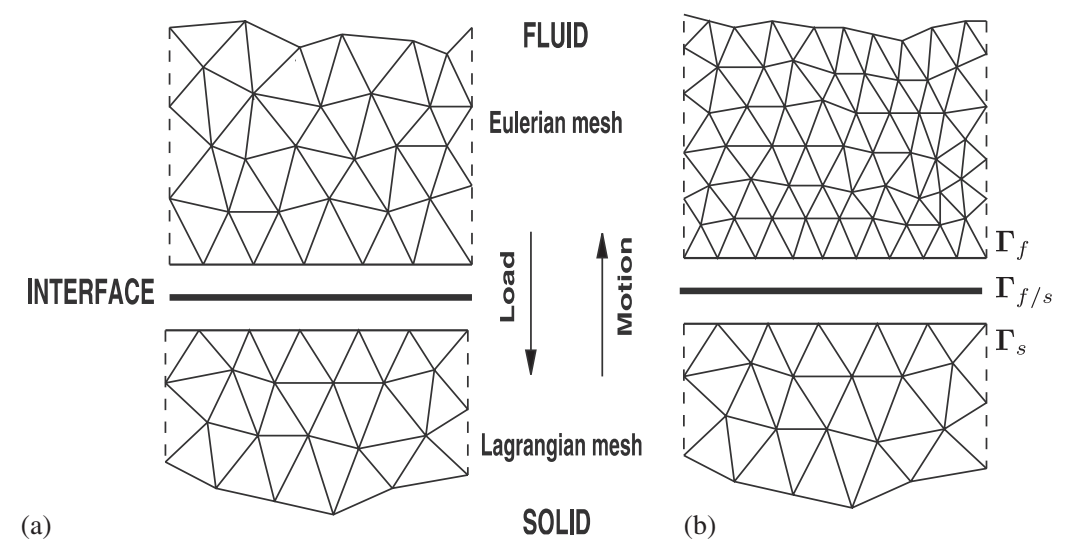

Figure 2. Unstructured planar interface meshes for coupled simulations:

(a) matching; and (b) non-matching.

\subsection{Discretization schemes}

The discretization of the load vector $\mathscr{R}_{\mathrm{s}}$ is more involved than that of $\mathscr{R}_{\mathrm{f}}$, because $\mathbf{t}_{\mathrm{f}}$ is defined on fluid boundary $\Gamma_{\mathrm{f}}$ while the shape function $N_{\mathrm{s}}$ is defined on the solid surface boundary $\Gamma_{\mathrm{s}}$. The interface may be matching (i.e. each nodal point of $\Gamma$ is shared by both the fluid side $\Gamma_{\mathrm{f}}$ and solid $\Gamma_{\mathrm{s}}$ ) or, more generally, non-matching (i.e. some of nodal points are not shared by the meshes of both domains, and $\Gamma_{\mathrm{f}}$ and $\Gamma_{\mathrm{s}}$ may not coincide with $\Gamma$ ). Figure 2 demonstrates the load and motion transfers between the fluid and solid solvers for matching and non-matching unstructured meshes.

2.3.1. Node-projection scheme. As proposed in Reference [4], this scheme projects the fluid nodes onto the solid surface element to extract the load vector on the solid interface nodes. Let $N_{\mathrm{s}}^{j}$ be a shape function associated with the node $j$ of the solid surface element $e$. This scheme can be outlined as follows:

- Associate each fluid node on $\Gamma_{\mathrm{f}}$ with the closest solid interface element on $\Gamma_{\mathrm{s}}$.

- Determine the projection of the fluid node onto the solid element.

- Multiply $\mathscr{R}_{\mathrm{f}}$ inside the solid element with $N_{\mathrm{s}}^{j}$.

The extracted load vector on the solid surface node is then

$$
\mathscr{R}_{\mathrm{s}}^{j}=\sum_{i=1}^{m_{\mathrm{f}}} N_{\mathrm{s}}^{j}\left(\mathbf{x}^{i}\right) \mathscr{R}_{\mathrm{f}}^{i}
$$

where $\mathbf{x}^{i}$ denotes the location of node $i$ of the fluid interface mesh. Figure 3 demonstrates this load transfer scheme using a 1-D interface consisting of six nodes on the fluid side (source mesh) and five nodes on the solid side (target mesh). From the summation property of shape functions, the nodal loads is conservative in the sense of Equation (4). 


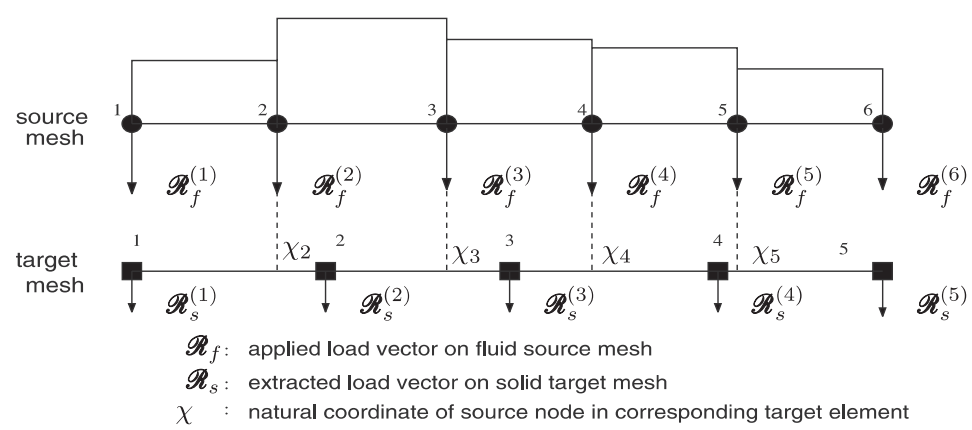

Figure 3. Illustration of node-projection based load transfer scheme.

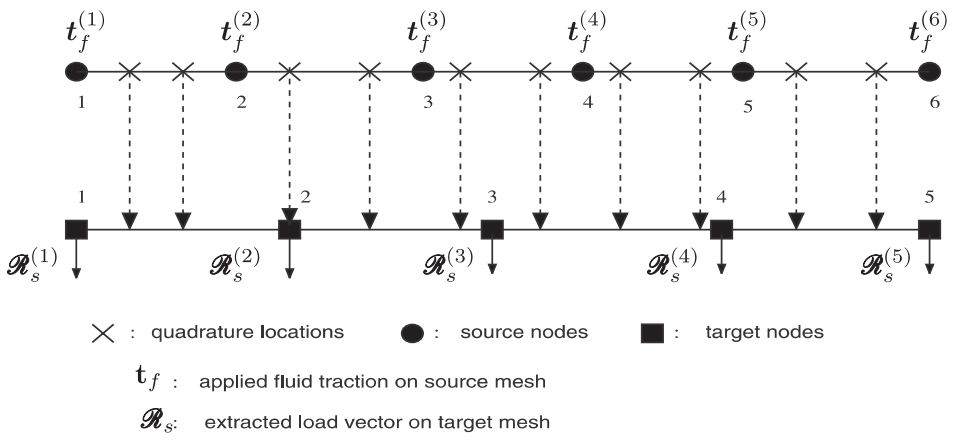

Figure 4. Illustration of quadrature-projection based load transfer scheme.

2.3.2. Quadrature-projection scheme. Similar to the node projection approach, this scheme projects the fluid quadrature points onto the solid surface element to extract $\mathbf{t}_{\mathrm{s}}$ and $\mathscr{R}_{\mathrm{s}}$ on the solid boundary [5]. An illustration of this scheme is given in Figure 4. It can be considered as a rough discretization of the Galerkin weighted residual method, where the integration is performed piecewise over the fluid interface elements with numerical quadrature. Specifically, $\mathscr{R}_{\mathrm{S}}$ is evaluated by

$$
\mathscr{R}_{\mathrm{s}}^{j}=\sum_{i=1}^{e_{\mathrm{f}}} \int_{\sigma_{\mathrm{f}}^{i}} N_{\mathrm{s}}^{j} \tilde{\mathbf{t}}_{\mathrm{f}} \mathrm{d} \Gamma
$$

where $e_{\mathrm{f}}$ denotes the number of elements on the fluid interface mesh, and $\sigma_{\mathrm{f}}^{i}$ denotes its $i$ th element. To avoid the situation in which a solid target element would receive no load, this scheme may rely on an adaptive quadrature rule by adaptively increasing the number of quadrature points. Further details about this load transfer scheme can be found in Reference [5].

The quadrature-projection scheme is also conservative in the sense of Equation (4). However, it can be very inaccurate due to a violation of the regularity assumption of the quadrature rules. In particular, given a solid node $j$ contained in the interior of a fluid element, the shape function $N_{\mathrm{s}}^{j}$ associated with the node is a hat function. Therefore, $N_{\mathrm{s}}^{j} \tilde{\mathbf{t}}_{\mathrm{f}}$ in Equation (11) 
exhibits discontinuity within the fluid element, and its integration using the quadrature points of the fluid element may involve arbitrarily large error. A similar inconsistency also applies to the node-projection scheme. Furthermore, the errors in these two schemes do not tend to zero as the input meshes are refined simultaneously, and hence they may result in non-convergence in grid refinement.

2.3.3. Common-refinement based scheme. In Reference [6], Jiao and Heath proposed a commonrefinement based scheme to resolve the violation of the regularity assumption. For piecewise constant functions, this scheme reduces to area-weighted averaging schemes in finite volume methods [13]. This scheme utilizes a common refinement, which is composed of the intersection of the elements of the fluid and solid meshes along the interface. This intersection defines subelements, as illustrated in Figure 5, over which the integration is performed. Algorithms for surface meshes are described in Reference [14], and this scheme in 2-D can be summarized as follows:

- Project the fluid interface nodes onto the solid mesh along the normal directions, where the normals are computed via element averaging.

- Sort the union of solid nodes and the projections of fluid nodes on the solid mesh to determine subelements on solid, and the inverse projections of fluid and solid nodes determine the corresponding subelements on the fluid mesh (as shown as the intermediate line in Figure 5).

- Integrate over the subelements using linear combination of fluid and solid geometry to compute $\mathscr{R}_{\mathrm{s}}$.

In this scheme, the load vector $\mathscr{R}_{\mathrm{s}}^{j}$ over the common refinement nodes is computed as

$$
\mathscr{R}_{\mathrm{s}}^{j}=\sum_{i=1}^{e_{\mathrm{c}}} \int_{\sigma_{\mathrm{c}}^{i}} N_{\mathrm{s}}^{j} \tilde{\mathbf{t}}_{\mathrm{f}} \mathrm{d} \Gamma
$$

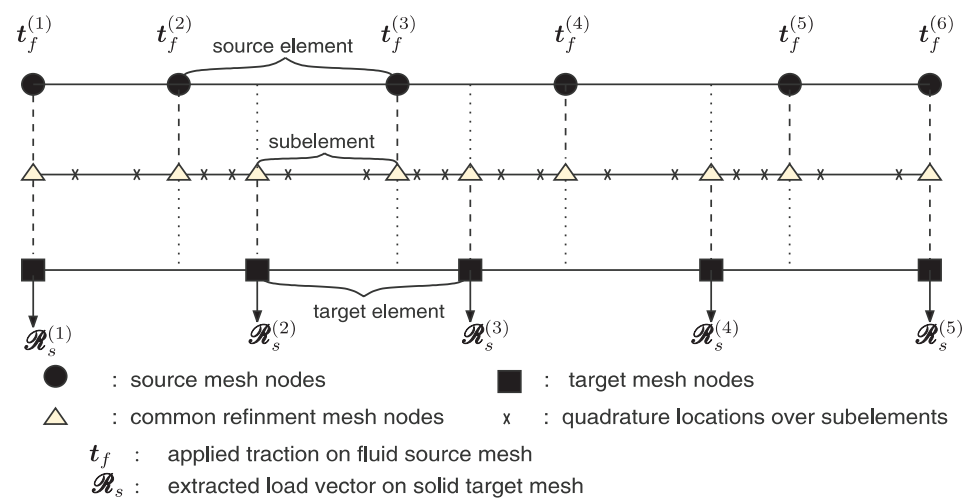

Figure 5. Illustration of common-refinement based load transfer scheme. 
where $e_{\mathrm{c}}$ denotes the number of subelements on the common refinement, and $\sigma_{\mathrm{c}}^{i}$ denotes its $i$ th subelement. As $N_{\mathrm{s}}$ and $N_{\mathrm{f}}$ are both low-degree polynomials within a subelement, the integration can be evaluated exactly using quadrature rules. To ensure optimal convergence and robustness, the quadrature rules should be sufficiently accurate to integrate the product $N_{\mathrm{s}} N_{\mathrm{f}}$ exactly.

Since the common-refinement based scheme minimizes the $L_{2}$ norm exactly, it is conservative and its discretization error is $O\left(h_{\mathrm{f}}^{2}+h_{\mathrm{s}}^{2}\right)$, where $h_{\mathrm{f}}$ and $h_{\mathrm{s}}$ are the mesh resolutions of fluid and solid meshes, respectively. Therefore, the scheme can transfer piecewise linear functions exactly [6]. By minimizing the Sobolev normal, the common-refinement based method can also handle strong gradients in the solution, unlike other higher-order interpolation methods which may experience spurious oscillations [6]. Computationally, the scheme is fairly inexpensive and easy to implement for curves.

\subsection{Time discretization and temporal coupling}

The fluid and solid solvers are integrated in time synchronously, thus there is a need for a continual transfer of load and motion at the interface. Therefore, a temporal coupling and updating is performed among fluid, mesh motion and solid solvers. It is likely that the time steps required by fluid and solid solvers are different, as the fluid and solid solvers generally employ different time integrators and different grid resolutions.

However, a common time step $\Delta t_{\mathrm{c}}$ is used herein to focus on spatial variation issues. This time step is determined by

$$
\Delta t_{\mathrm{c}}=\min \left(\Delta t_{\mathrm{f}}, \Delta t_{\mathrm{s}}\right)
$$

where $\Delta t_{\mathrm{f}}$ and $\Delta t_{\mathrm{s}}$ are fluid and solid time steps, respectively, and are restricted by their respective Courant-Friedrichs-Levy (CFL) conditions.

Assuming that the fluid, solid and mesh motion solutions are known at time $t^{n}$, we obtain the solution of the coupled FSI system at time $t^{n+1}$ using the following explicit coupling procedure:

1. Predict the state (i.e. displacement) of the solid at the end of the current time step $\left(t^{n+1}\right)$.

2. Calculate the mesh movement during the current time step.

3. Integrate the fluid system to the next time level.

4. Update the solid to next time level using the load vector on the interface.

To assess the accuracy associated with the three conservative load transfer schemes, we turn our attention next to four test problems of increasing complexity: the first problem involves a static planar interface with varying grid resolutions and other three transient fluid-solid interaction problems with the deforming interfaces. As we mentioned earlier, for simplicity, our experiments focus on 2-D FSI problems. The comparative assessment of the precision of the three load transfer schemes is however expected to generalize to higher dimensions.

\section{STATIC ANALYSIS OF LOAD TRANSFER SCHEMES}

The objective of this study is to assess systematically the sensitivity and accuracy of the load transfer schemes with respect to the mesh resolutions and mismatches. We transfer tractions on the fluid (source) interface mesh $\Gamma_{\mathrm{f}}$ to nodal forces at the solid (target) interface mesh $\Gamma_{\mathrm{s}}$, where 
the geometry of the interface is flat. A range of mesh mismatches are obtained by varying the ratio of number of source (fluid) and target (solid) elements (with matching end points of $\Gamma_{\mathrm{f}}$ and $\Gamma_{\mathrm{S}}$ ). A pressure profile is prescribed analytically over the interface to have either constant, linear, or quadratic functions on $\Gamma_{\mathrm{f}}$. The number of nodes of the fine mesh is fixed, while that of the coarse mesh vary. To normalize the range of the relative grid resolution of fluid $\left(h_{\mathrm{f}}\right)$ and solid $\left(h_{\mathrm{s}}\right)$ meshes for plotting purpose, we define a mesh ratio parameter $\gamma_{\mathrm{m}}$ as

$$
\gamma_{\mathrm{m}}=\frac{1-\left(h_{\mathrm{f}} / h_{\mathrm{s}}\right)}{1+\left(h_{\mathrm{f}} / h_{\mathrm{s}}\right)}
$$

which ranges between -1 and 0 if the solid mesh is finer and ranges between 0 and 1 otherwise.

The shape functions are assumed to be linear, so the approximation of pressure on $\Gamma_{\mathrm{f}}$ is piecewise linear. We compute relative errors in the load vector $\mathscr{R}_{\mathrm{s}}$ as

$$
\varepsilon_{l}=\frac{\left\|\mathscr{R}_{\mathrm{s}}^{\text {numerical }}-\mathscr{R}_{\mathrm{s}}^{\text {exact }}\right\|_{2}}{\left\|\mathscr{R}_{\mathrm{s}}^{\text {exact }}\right\|_{2}}
$$

where $\mathscr{R}_{\mathrm{s}}^{\text {exact }}$ is computed over $\Gamma_{\mathrm{s}}$ from the analytical pressure profile, and $\mathscr{R}_{\mathrm{s}}^{\text {numerical }}$ is obtained from one of the load transfer schemes. If the pressure over $\Gamma_{\mathrm{s}}$ was obtained by solving Equation (7), then relative errors in the pressure could be defined similarly.

Figure 6 shows the dependence of the error $\varepsilon_{l}$ in $\mathscr{R}_{\mathrm{s}}$ on the mesh ratio parameter $\gamma_{\mathrm{m}}$ for a linear pressure profile with 41 nodes on the fine mesh. The common-refinement based scheme performs consistently well for all the mesh mismatch cases, with an error near the machine precision. For the node- and quadrature-projection schemes, on the other hand, more than 12 orders of magnitude larger errors are observed for almost all cases. The errors in the latter

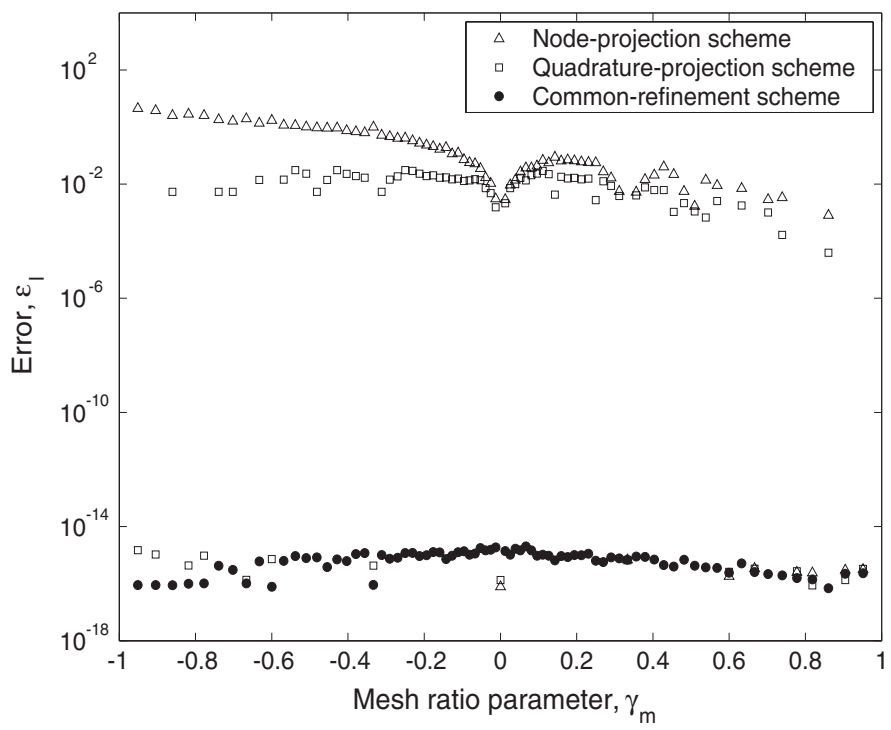

Figure 6. Dependence of load vector error on relative mesh parameter $\gamma_{\mathrm{m}}$ for the three load transfer schemes. 

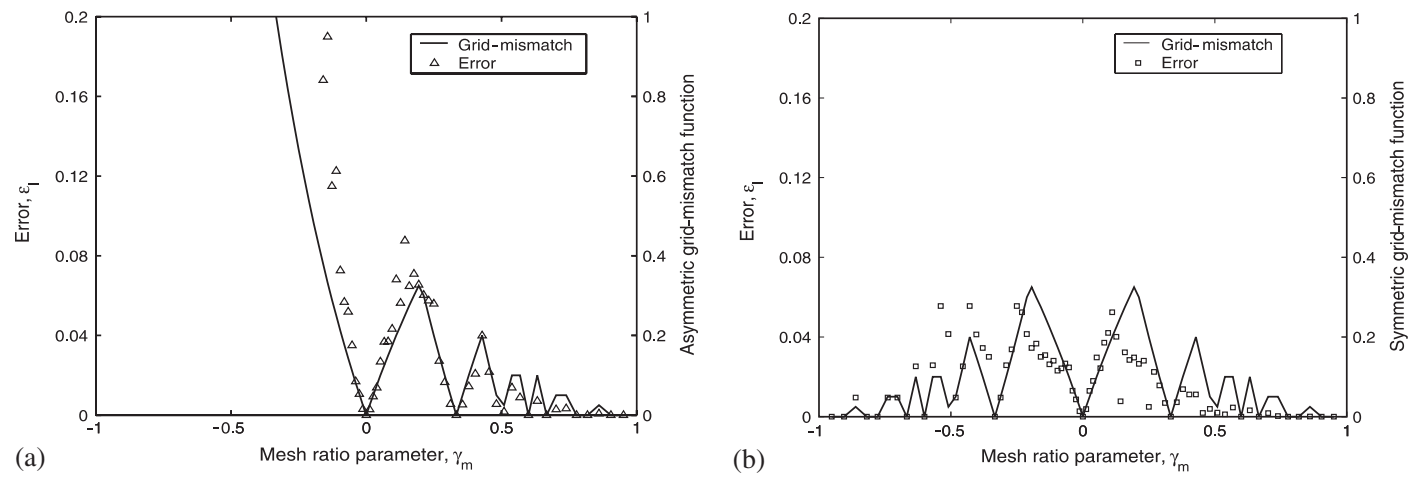

Figure 7. Correlation between load vector error and grid mismatch function $\operatorname{gmm}\left(h_{\mathrm{f}}, h_{\mathrm{s}}\right)$ for: (a) node-projection scheme; and (b) quadrature-projection scheme.

two schemes oscillate drastically with respect to $\gamma_{\mathrm{m}}$ and are at near machine precision at isolated points when the fine mesh is nested in the coarse mesh. Similar error profiles were obtained when using quadratic, and even constant pressure profiles on the source mesh. In a mesh refinement study using 81 and 161 nodes for finer meshes, the errors in node- and quadrature-projection schemes show the same trend and have the same orders of magnitude, demonstrating that errors in these schemes do not tend to zero as input meshes are refined simultaneously. Similar results were obtained using other error measures.

As stated earlier, the reason of large errors in the node- and quadrature-projection schemes is the violation of regularity assumption of the quadrature rules. To verify this claim and characterize the oscillatory patterns in the errors, we define a grid-mismatch function

$$
\operatorname{gmm}\left(h_{1}, h_{2}\right)=\left|\max \left\{\operatorname{nint}\left(\frac{h_{1}}{h_{2}}\right) \times\left(\frac{h_{2}}{h_{1}}\right),\left(\frac{h_{2}}{h_{1}}\right)\right\}-1\right|
$$

where the nint function rounds a real number to its nearest integer. The gmm function for a given mesh measures how close the ratio between $h_{1}$ and $h_{2}$ is to a positive integer, and a larger value of gmm would, in general, correspond to a more severe violation of the regularity assumption.

For the node-projection scheme, a strong correlation between the relative error with $\operatorname{gmm}\left(h_{\mathrm{f}}, h_{\mathrm{s}}\right)$ exists, as demonstrated in Figure 7(a), where the peaks and dips of errors follow those of this grid-mismatch function. For the quadrature-projection scheme, which applies an adaptive quadrature rule when the fluid (source) mesh is coarse, the errors correlate to a symmetric grid-mismatch function, $\min \left\{\operatorname{gmm}\left(h_{\mathrm{f}}, h_{\mathrm{s}}\right), \operatorname{gmm}\left(h_{\mathrm{s}}, h_{\mathrm{f}}\right)\right\}$, as shown in Figure $7(\mathrm{~b})$. The strong correlations between the errors in node- and quadrature-projection schemes and the grid-mismatch functions relate the sensitivity of these methods to the grid mismatch, and will help to discuss errors in following FSI problems.

\section{ELASTIC PISTON PROBLEM}

To evaluate the integrated framework for transient fluid-solid interaction, the accuracy of the load transfer schemes are compared for three test problems involving the coupling of an 
inviscid fluid with a solid, where the interface is assumed to be impermeable, non-reactive and adiabatic. With these assumptions, the interface can be treated as a contact discontinuity with the following properties: (a) no mass flux; (b) no jump in normal velocity; (c) free-slip boundary condition for the tangential velocity; (d) no jump in the normal stress. However, in these problems, jumps in density and entropy across the discrete fluid-solid interface are allowed. All the above conditions and treatments are enforced by the interface coupling schemes through the application of boundary conditions at discrete times.

The first comparative assessment involves the analysis of a transient quasi 1-D FSI problem consisting of expansion fans in the fluid domain coupled with a compression wave in the elastic material domain. This flexible piston problem has a 1-D analytical solution for an inviscid compressible flow and linearly elastic solid. The closed-form solution is used to verify the coupling of fluid-solid interaction as well as for the accuracy analysis of the load transfer schemes. Figure 8 shows the expansion fans associated with the movement of an interface of elastic piston lying between rollers. Initially, the fluid domain has quiescent inviscid compressible fluid. It is assumed that there is no reflection of expansion fans in the fluid domain and compression wave in the solid domain.

We use a perfect gas $(\gamma=1.4)$ with the physical parameters, an initial density $\rho_{0}=$ $8.75 \mathrm{~kg} / \mathrm{m}^{3}$, and an initial pressure $p_{0}=100 \mathrm{MPa}$. For the elastic solid, we choose properties similar to that of aluminum, with Poisson's ratio $v=0.29$, density $\rho_{\mathrm{s}}=2800 \mathrm{~kg} / \mathrm{m}^{3}$, and Young's modulus $E=70 \mathrm{GPa}$. From these properties, we obtain the speed of sound $c_{0}=\sqrt{\gamma p_{0} / \rho_{0}}=4000 \mathrm{~m} / \mathrm{s}$ in the fluid and the dilatational wave speed $c_{\mathrm{d}}=\sqrt{E / \rho_{\mathrm{s}}\left(1-v^{2}\right)}=$ $5224 \mathrm{~m} / \mathrm{s}$ in the solid domain for plane stress assumption. We consider the entire coupled system to be quiescent and stress free at $t=0$ for the fluid and solid, respectively. Figure 8

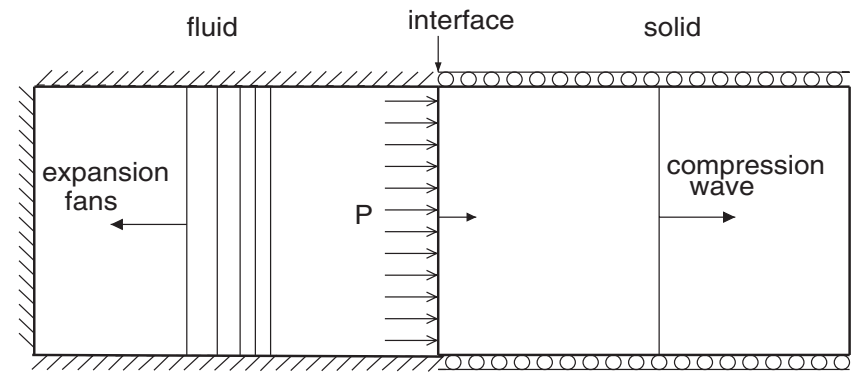

(a)
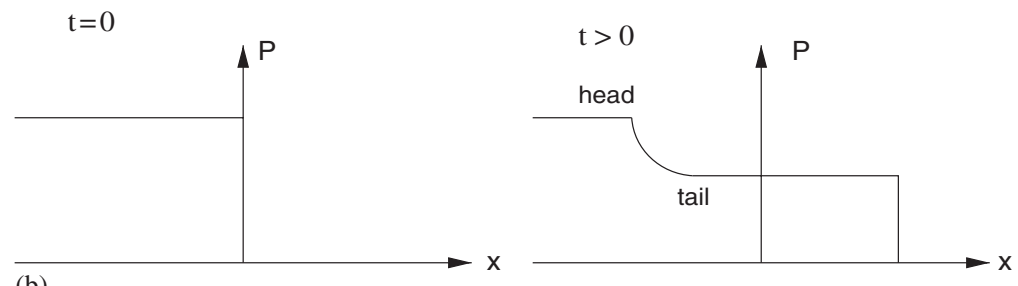

Figure 8. (a) Schematic of elastic piston problem; and (b) pressure as function of $x$ for simple centered expansion fans in fluid domain and compression wave in solid domain. 
shows the computational domain for this problem. The Eulerian fluid domain is closed by slip walls other than solid interface boundary. The Lagrangian solid domain has roller boundary condition on the top and bottom walls and traction free condition along the vertical end.

Figure 9 shows the solution of velocity field for expansion fans in the fluid domain and a compression wave in the solid domain obtained with matching meshes. Note that the result is effectively one dimensional in the fluid and solid domains. Furthermore, the fluid properties before, after, and in the expansion fans and the solutions of solid domain and of interface velocity are consistent with the analytical solution.

Next, we analyse the effects of non-matching load transfer schemes in the interface solution (displacement and velocity) of solid surface, using disparate meshes across the fluid-solid interface. Figure 10 shows two pairs of representative meshes used in this study, where the resolution of the fluid mesh $\left(h_{\mathrm{f}}\right)$ is fixed and the resolution of solid mesh $\left(h_{\mathrm{s}}\right)$ is varied to obtain various mesh mismatches.

Although the theoretical solution is 1-D, with an interface front moving at a uniform speed, the non-matching interface meshes yield spatial variations and in the local interface velocity in the $x$ direction $\left(\dot{u}_{x, I}\right)$. We evaluate the spatial non-uniformity of the velocity from the interface averaged solution $\left(\dot{u}_{x, I}^{\text {averaged }}\right)$ over the range of time evolution as

$$
\varepsilon_{\mathrm{V}}=\frac{\left\|\dot{u}_{x, I}-\dot{u}_{x, I}^{\text {averaged }}\right\|_{2}}{\left\|\dot{u}_{x, I}^{\text {averaged }}\right\|_{2}}
$$

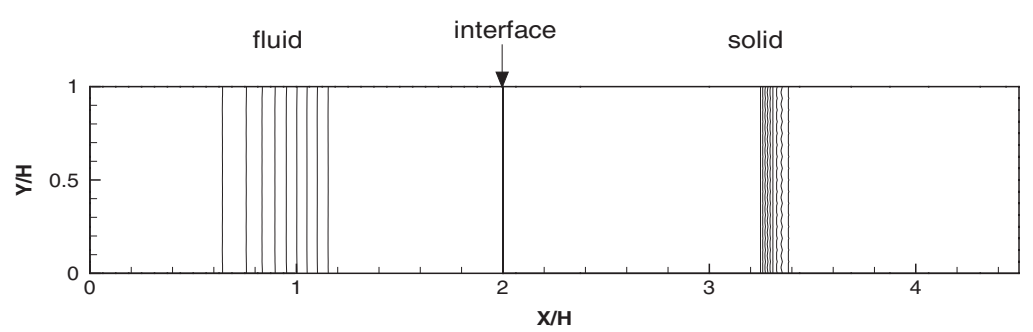

Figure 9. Contour plot of calculated velocity field for the elastic piston problem with matching interface meshes.

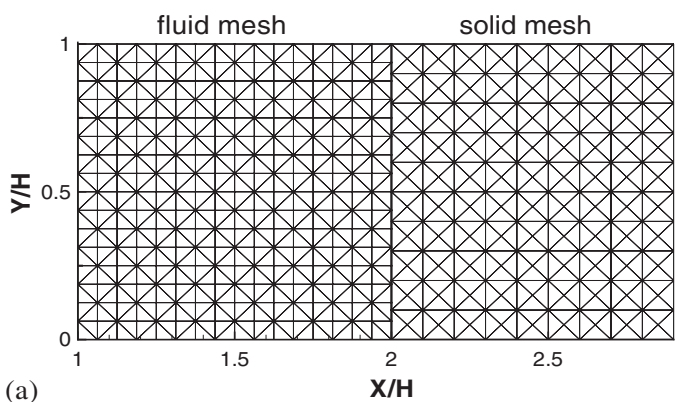

(a) $\mathrm{X} / \mathrm{H}$

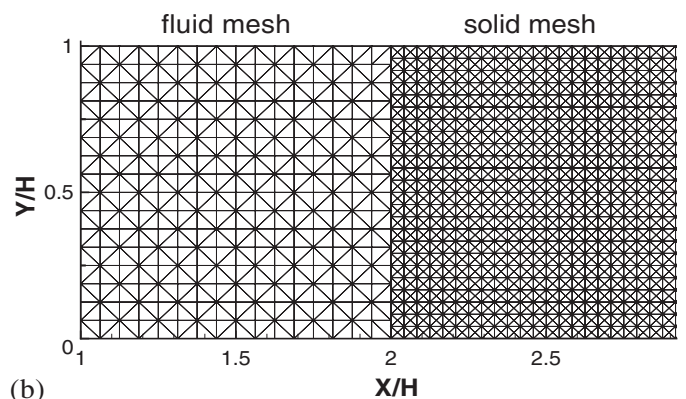

(b)

Figure 10. Representative meshes for the elastic piston problem: (a) fine-to-coarse projection case $\left(h_{\mathrm{f}} / h_{\mathrm{S}}\right)=0.625$; and (b) coarse-to-fine projection case $\left(h_{\mathrm{f}} / h_{\mathrm{s}}\right)=1.5$. 
Similarly, the non-uniformity in the $x$ component of the interface displacement $\left(u_{x, I}\right)$ from the averaged interface displacement $\left(u_{x, I}^{\text {averaged }}\right)$ is evaluated as

$$
\varepsilon_{\mathrm{d}}=\frac{\left\|u_{x, I}-u_{x, I}^{\text {averaged }}\right\|_{2}}{\left\|u_{x, I}^{\text {averaged }}\right\|_{2}}
$$

Figure 11 depicts the evolution $\varepsilon_{\mathrm{V}}$ and $\varepsilon_{\mathrm{d}}$ for the repeated load transfer in fine-to-coarse projections with $h_{\mathrm{f}} / h_{\mathrm{S}}=0.875$ and 0.5 , respectively, with the time normalized by the time travel of the dilatational wave speed $c_{\mathrm{d}}$ along the interface height $H$. It is apparent that in the case of $h_{\mathrm{f}} / h_{\mathrm{s}}=0.875$, significantly larger non-uniformity errors exist in the interface velocity and displacement for the traditional schemes compared to the common-refinement based scheme, especially for the quadrature-projection scheme. In the case of $h_{\mathrm{f}} / h_{\mathrm{s}}=0.5$, where the grid
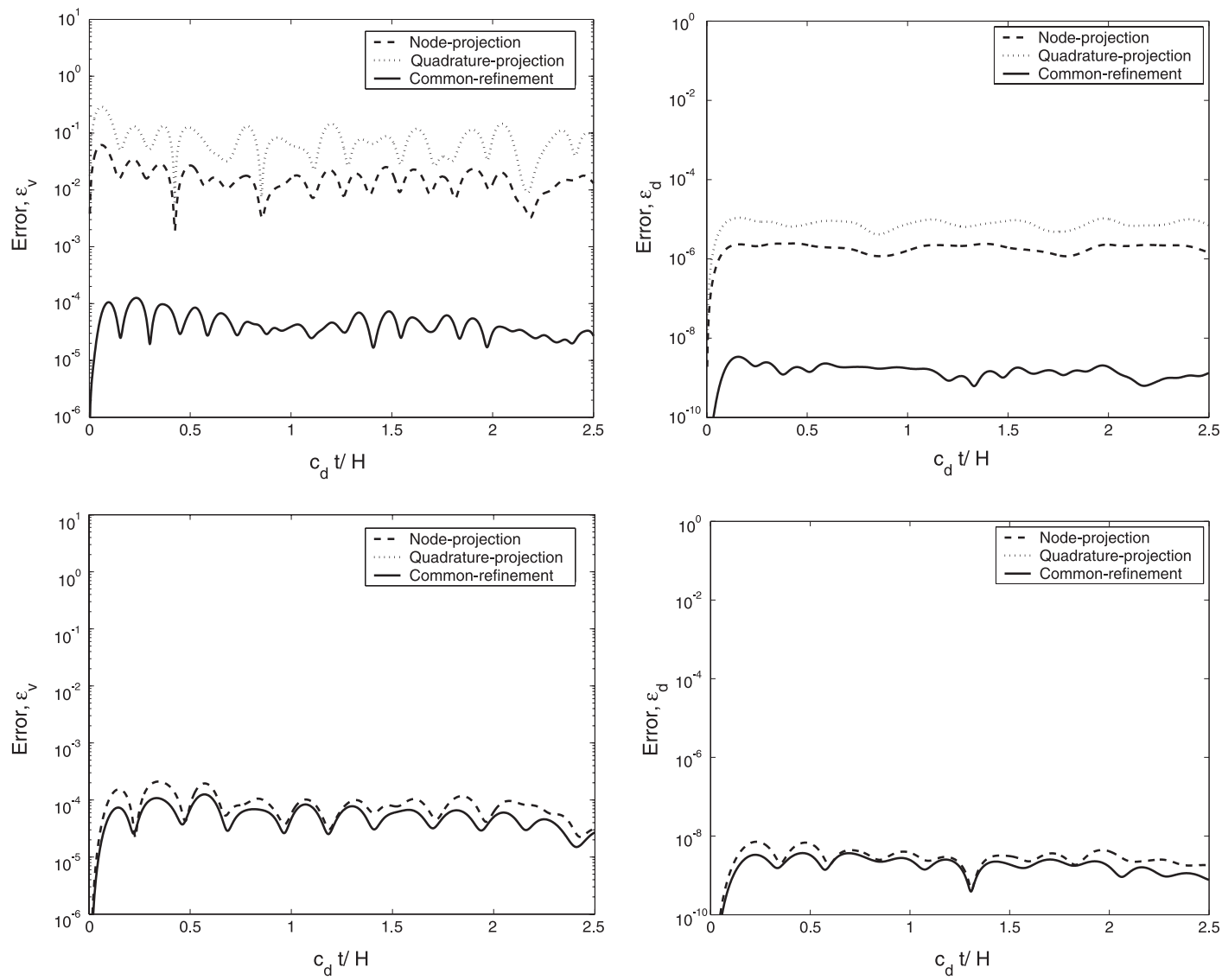

Figure 11. Evolution of non-uniformity in interface velocity $\left(\varepsilon_{\mathrm{v}}\right)$ and displacement $\left(\varepsilon_{\mathrm{d}}\right)$ obtained for the elastic piston problem with the three load transfer schemes. The top figures correspond to $h_{\mathrm{f}} / h_{\mathrm{s}}=0.875$ and the bottom figures to $h_{\mathrm{f}} / h_{\mathrm{s}}=0.5$. 
mismatch function is 0 (i.e. each solid node has a matching fluid node), all three load transfer schemes give the same non-uniformity errors in the velocity and displacement as expected.

Figure 12 depicts the non-uniformity in the interface velocity and displacement in the cases of two coarse-to-fine projections with $h_{\mathrm{f}} / h_{\mathrm{s}}=1.125$ and 1.5. Similar to the previous mismatched fine-to-coarse results, the case of $h_{\mathrm{f}} / h_{\mathrm{s}}=1.125$ shows significantly larger nonuniformity errors for the traditional schemes compared to the common-refinement based scheme. The case of $h_{\mathrm{f}} / h_{\mathrm{s}}=1.5$ also suffers from larger oscillatory errors and instability for the nodeprojection scheme due to severe violation of regularity assumption of the quadrature rules. The common-refinement based scheme, on the other hand, has orders of magnitude smaller errors in both cases.

Furthermore, we observe the dependence of time-averaged interface non-uniformity errors on the mesh mismatch similar to the 1-D static study. To obtain a time-averaged measure, we
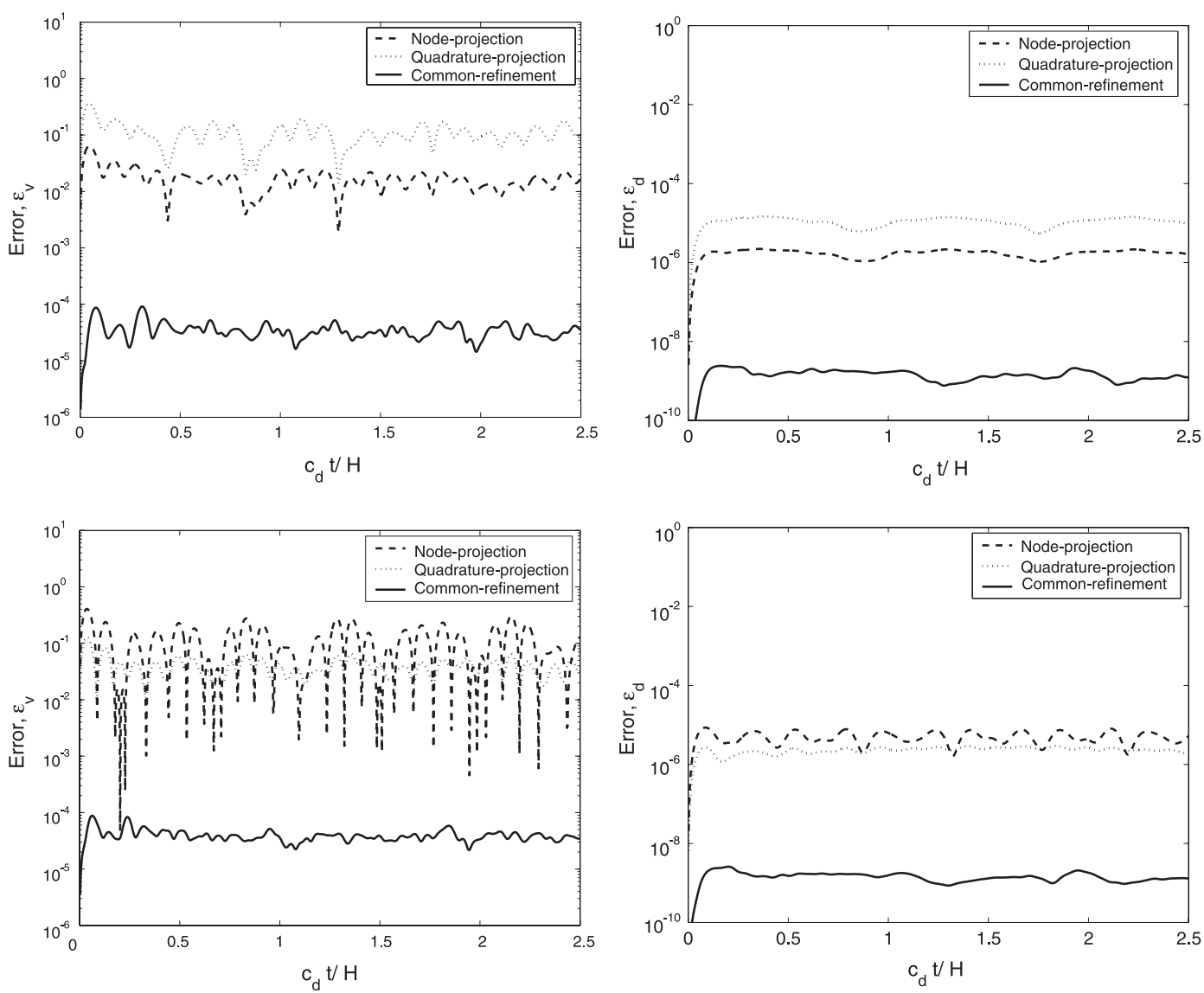

Figure 12. Evolution of non-uniformity in interface velocity $\left(\varepsilon_{\mathrm{V}}\right)$ and displacement $\left(\varepsilon_{\mathrm{d}}\right)$ obtained for the elastic piston problem with the three load transfer schemes. The top figures correspond to $h_{\mathrm{f}} / h_{\mathrm{s}}=1.125$ and the bottom figures to $h_{\mathrm{f}} / h_{\mathrm{s}}=1.5$. 

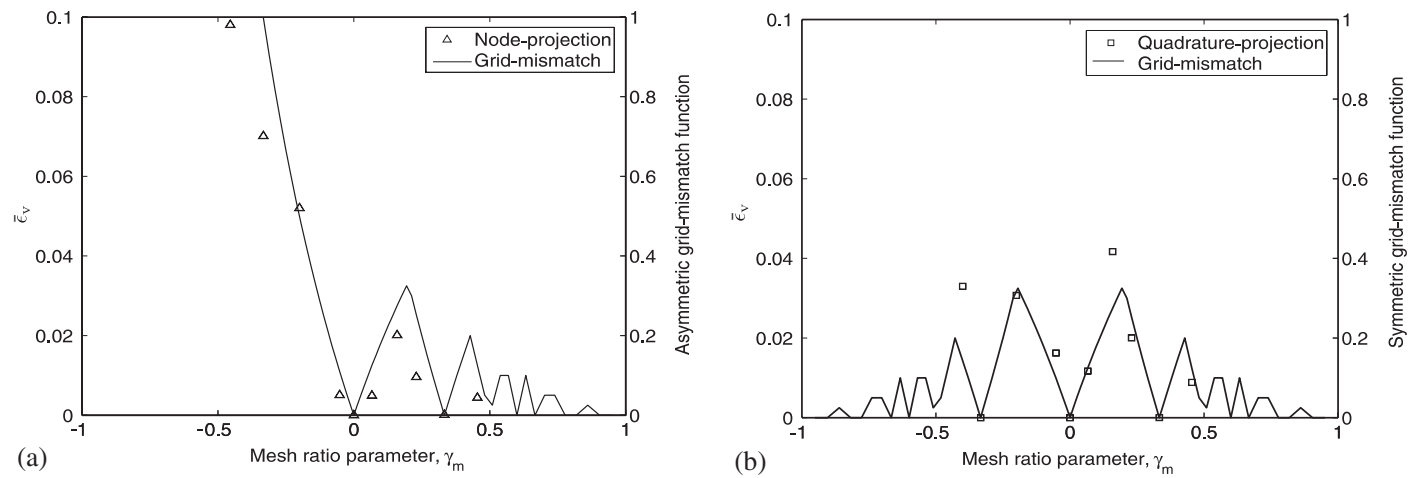

Figure 13. Dependence of time-averaged interface velocity error $\bar{\varepsilon}_{\mathrm{V}}$ on mesh mismatch and correlation with grid mismatch function, where the symbols correspond to numerical values obtained for the elastic piston problem: (a) node-projection scheme; and (b) quadrature-projection scheme.

integrate the evolution profiles of interface velocity non-uniformity (see Figures 11 and 12) of the load transfer schemes. Thus, the time-averaged error of the interface velocity $\bar{\varepsilon}_{\mathrm{v}}$ is given by

$$
\bar{\varepsilon}_{\mathrm{V}}=\frac{\int_{t^{*}=0}^{T} \varepsilon_{\mathrm{V}} \mathrm{d} t^{*}}{\int_{t^{*}=0}^{T} \mathrm{~d} t^{*}}
$$

where $t^{*} \equiv c_{\mathrm{d}} t / H$ is a non-dimensional time scale, and $T$ is equal to 2.5 in the present calculation. Figure 13 shows correlations between grid-mismatch functions and the time-averaged non-uniformity errors for the node- and quadrature-projection schemes. These correlations are consistent with the 1-D static analysis presented in Section 3 indicating the appropriateness of gmm to explain such errors. Similarly, the time-averaged error for the common-refinement based scheme is less than $10^{-4}$ and thus several orders of magnitude smaller than these two schemes as noted previously, and is nearly zero in the scale used in the plots in Figure 13.

\section{SUPERSEISMIC SHOCK PROBLEM}

In another comparative study between the three load transfer schemes, we simulated the 2-D fluid-solid interaction problem consisting of a shock in a compressible fluid traveling at a superseismic speed over an elastic half space, i.e. at a speed $V_{\mathrm{sh}}$ that exceeds the dilatational wave speed of the solid (Figure 14(a)). This problem presents an interesting coupling between fluid and solid solutions since the deformation of the elastic solid behind the traveling shock affects the fluid flow by changing the shock angle and thereby the intensity of the pressure jump across the shock. Furthermore, this problem is also chosen because it has an analytical similarity solution in the frame of the traveling shock and thereby constitutes an excellent test problem for the explicit 2-D FSI framework described in earlier sections and, in particular, the interface transfer schemes. This problem was first investigated analytically by Bleich [15], who studied the coupled wave systems created by superseismic load over elastic and granular material. It was also used in Reference [16] to test the accuracy of a level set scheme for coupled 


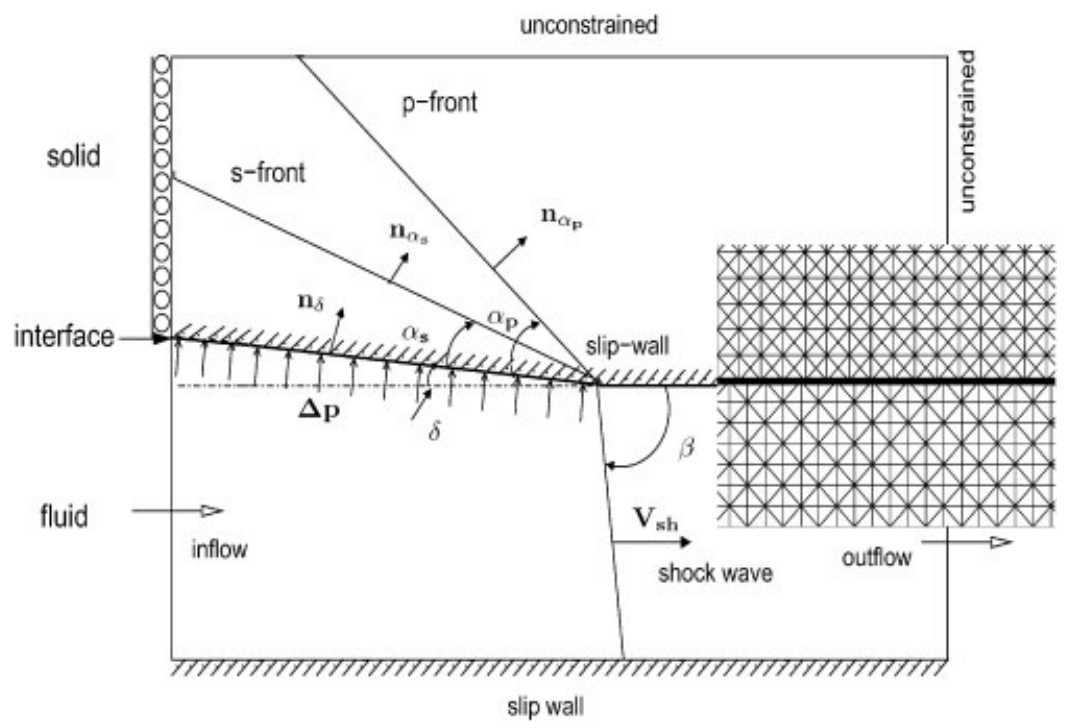

Figure 14. Schematic and computational domain of superseismic shock problem, and details of the non-matching meshes near the interface.

simulations using matching meshes. For completeness, the analytical solution is summarized in Appendix A.

In this verification study, we select the fluid flow and solid material properties such that the acoustic impedances of the two media are comparable and a significant superseismic coupling occurs. For the linear elastic solid, we use the properties similar to those of copper: Poisson's ratio $v=0.33$, density $\rho_{\mathrm{s}}=8970 \mathrm{~kg} / \mathrm{m}^{3}$, and Young's modulus $E=110 \mathrm{GPa}$. For the fluid, we use a perfect gas with an artificially high initial density, $\rho_{1}=1033 \mathrm{~kg} / \mathrm{m}^{3}$, and an initial pressure $p_{1}=5.55 \mathrm{GPa}$. The initial upstream Mach number for this problem is $M_{1}=2.952$. We consider the unshocked fluid system to be under initial pressure $p_{1}$ and solid domain to stress free. The net traction along the interface, $\Delta p$, is computed by subtracting $p_{1}$ from the calculated Eulerian fluid pressure field behind the shock $p_{2}$ (see Appendix A). This yields a zero applied pressure load in the undisturbed fluid region. As indicated in Figure 14, the boundary conditions for the fluid solver are supersonic inflow on the left side of the domain, and along the mesh boundaries that are not in contact with the fluid, the solid domain has unconstrained and roller boundary conditions. Figure 14 also depicts the non-matching meshes for the fluid and solid domains along the interface. We have chosen uniform triangular meshes to avoid any numerical oscillations caused by the finite volume and the finite element discretizations in the fluid and solid domains, respectively.

The coupled problem is then solved first with a sufficiently resolved matching interface, for which the three load transfer schemes yield the same solution, and then for non-matching fluid and solid meshes. Special emphasis is placed on the resulting deformed shape of the interface and on its effect on the fluid and solid solution in the vicinity of the fluid-solid interface. Typical numerical solutions obtained with matching and non-matching meshes are presented in Figure 15 , clearly showing the normal shock propagating in the fluid domain, the $\mathrm{p}$ - and s-wave 

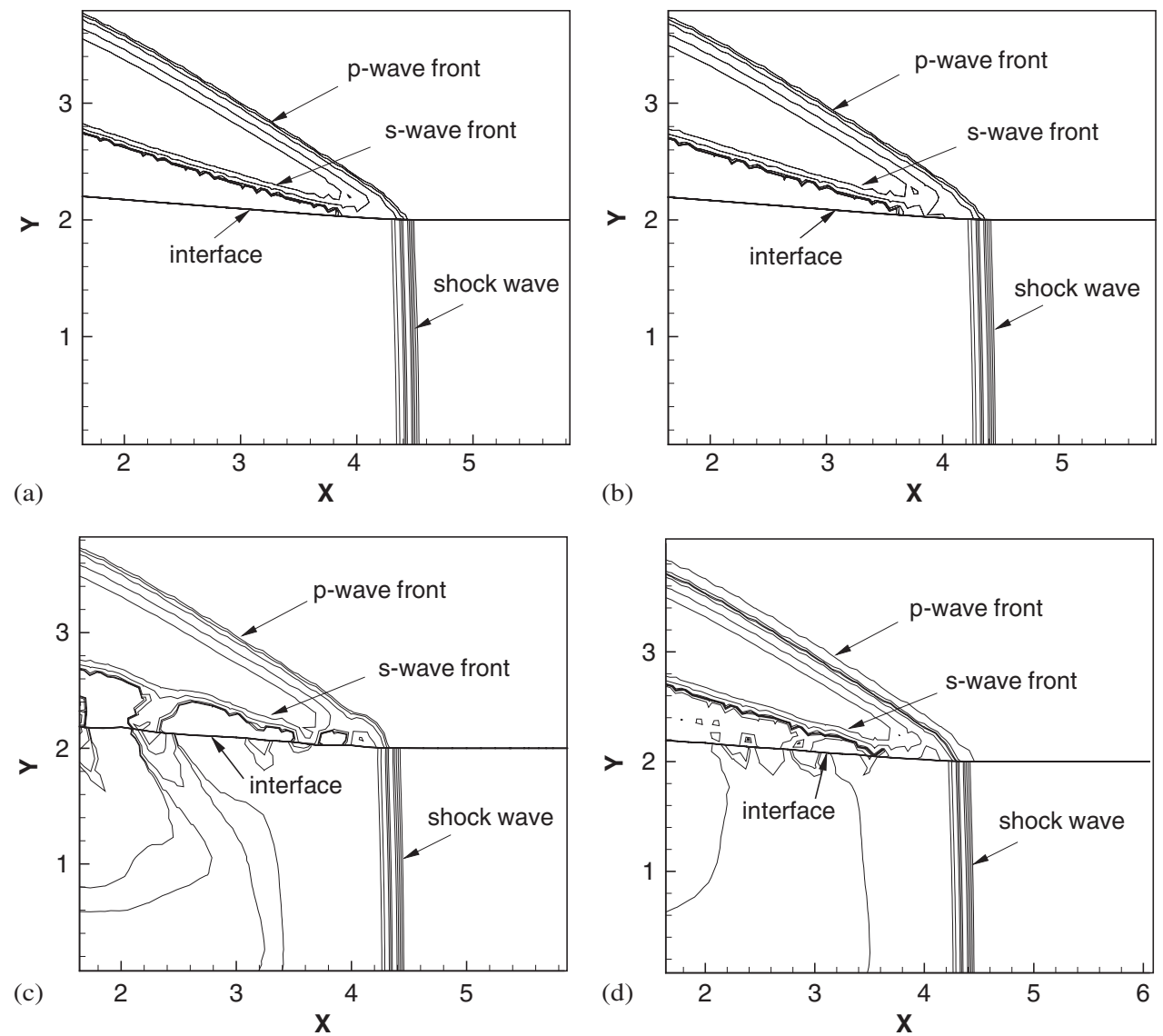

Figure 15. Superseismic shock problem: contours of pressure in the fluid domain and shear stress in the solid domain for various load transfer schemes: (a) matching meshes $\left(h_{\mathrm{f}} / h_{\mathrm{s}}=1.0\right)$; (b) common-refinement based scheme; (c) quadrature-projection scheme; and (d) node-projection scheme. In (b)-(d), the interface meshes do not match and $h_{\mathrm{f}} / h_{\mathrm{s}}=0.875$.

'Mach cones' in the solid domain, and the deformation of the fluid-solid interface behind the shock. Note that in the analytical solution, the interface is predicted to remain straight with an angle that depends on the shock speed and the material properties. Figure 15(a) presents contour plots of the pressure distribution in the lower fluid domain and of the shear stress distribution in the upper solid domain for the matching interface meshes. Again, the three load transfer schemes yield the same results for the matching meshes and these compare well with the analytical solution. The analytical and numerical values of the various angles entering this problem, as defined in Figure 14, are presented in Table I for the case of matching interface meshes. The solution with the matching interface is used as the reference numerical solution achievable with the explicit FSI scheme for the given spatial and temporal discretizations.

Figures $15(\mathrm{~b})-(\mathrm{d})$ present contour plots obtained with the three load transfer schemes using the same spatial and temporal discretization for a non-matching interface with $h_{\mathrm{f}} / h_{\mathrm{s}}=0.875$ 
Table I. Analytical and numerical angles of the superseismic shock problem with matching meshes.

\begin{tabular}{lccc}
\hline Variable & Analytical (deg) & Numerical (deg) & Error (approx) (\%) \\
\hline$\delta$ & 4.23 & $4.14^{\circ} \pm 0.04^{\circ}$ & 2.36 \\
$\alpha_{\mathrm{s}}$ & 11.13 & $11.36^{\circ} \pm 0.09^{\circ}$ & 2.06 \\
$\alpha_{\mathrm{p}}$ & 27.49 & $28.16^{\circ} \pm 0.12^{\circ}$ & 2.43 \\
$\beta$ & 1.71 & $1.73^{\circ} \pm 0.08^{\circ}$ & 1.11 \\
\hline
\end{tabular}
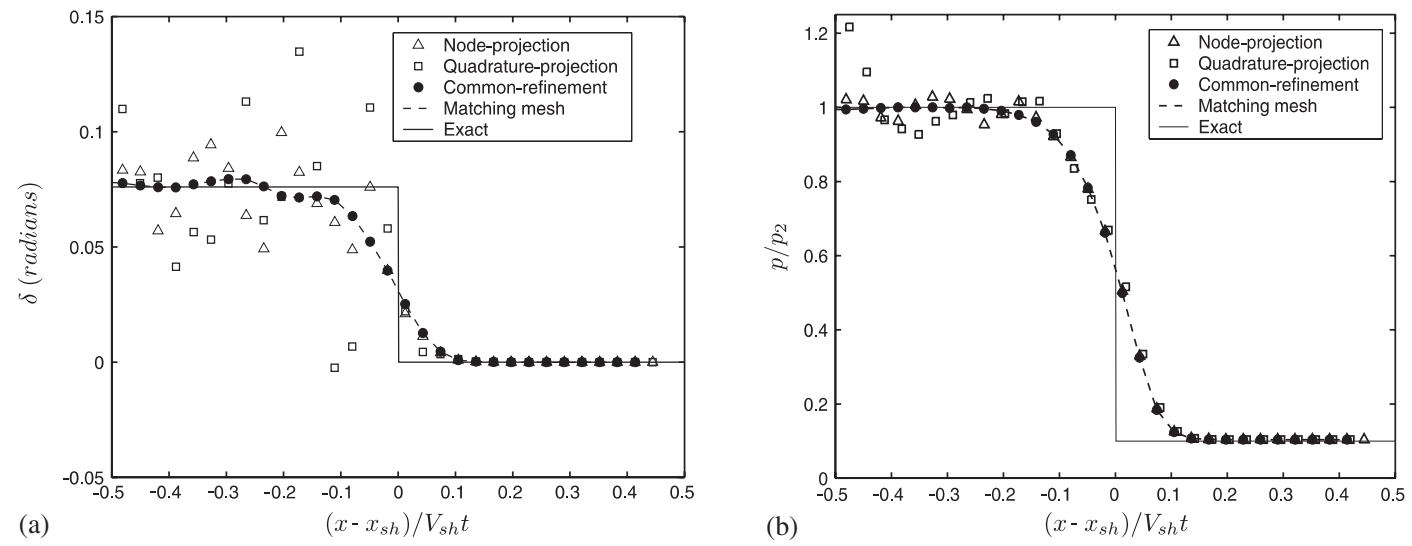

Figure 16. Comparison of interface and fluid solutions: (a) deflection angle $\delta$; and (b) pressure $p$ along the fluid boundary. $x_{\text {sh }}$ and $V_{\text {sh }}$ denote the theoretical shock position and speed, respectively.

(i.e. $\left.\gamma_{\mathrm{m}}=0.067\right)$. Figure $15(\mathrm{~b})$ corresponds to the common-refinement based load transfer scheme. For this load transfer scheme, the interface deflection is straight, and the fluid and solid solutions are quite uniform and similar to matching interface solutions. On the other hand, the results shown in Figures 15(c) and (d) exhibit significant spurious oscillations along the interface due to the inaccurate load transfer by quadrature- and node-projection schemes, respectively. Such errors and oscillations are especially prominent with the quadrature-projection scheme, as also observed in the elastic piston problem.

Figure 16(a) shows the spatial variation of the interface deflection angle in radians of the fluid-solid interface for the non-matching meshes, using the matching-mesh and exact solutions as references. This angle is calculated using the slope of piecewise linear segments. We observe substantial errors in the interface displacement for the traditional load transfer schemes, while the common-refinement based scheme yields a uniform and smooth interface displacement very similar to the matching mesh solution. Furthermore, inaccuracy in the load transfer not only affects the deformed shape of the interface, but also the solution in the fluid and solid domains.

This effect is quantified in Figure 16(b), which shows a comparison between the analytical and numerical distributions of the normalized fluid pressure $p / p_{2}$ along the fluid-solid interface. The numerical values have been obtained for matching and non-matching meshes with the three 
load transfer schemes. As expected, in all cases, the numerical solutions tend to smoothen the shock at $x \approx x_{\mathrm{sh}}$, where $x_{\mathrm{sh}}$ denotes the theoretical shock location. The pressure rise is 'smeared' over the 5 or 6 elements (see Figure 16(b)). However, the figure clearly illustrates the oscillatory nature of the solution further behind the shock $\left(x<x_{\mathrm{sh}}\right)$ obtained with the two traditional load transfer schemes, with errors reaching up to $20 \%$. The common-refinement based scheme, however, yields results almost identical to those obtained with matching meshes. We have also obtained similar findings for the normalized shear stress distribution along the interface of the solid domain.

\section{FLEXIBLE INHIBITOR PROBLEM}

To assess the effects of load transfer schemes on aeroelastic problems involving large displacements, we now consider the aeroelastic deformations of a flexible beam subjected to a subsonic flow field. This problem is motivated by that of an inhibitor protruding into the core flow field of a solid rocket motor [17,18].

The problem geometry is illustrated in Figure 17 which presents the meshes in the fluid and solid domains. The number of interface nodes is the same for both meshes, but the nodal positions differ along the fluid-solid interface for the non-matching meshes. To capture the bending response of the beam, six-node triangular elements that include a non-linear kinematic description for large displacement and rotations are used in the solid domain. The fluid domain has a relatively coarse mesh, and viscosity is neglected in this calculation. The selected fluid flow and solid material properties lead to a substantial deflection of the beam. For the elastic solid beam, we use isotropic properties similar to those of the solid propellant: Poisson's ratio $v=0.499$, density $\rho_{\mathrm{s}}=1300 \mathrm{~kg} / \mathrm{m}^{3}$, and Young's modulus $E=10 \mathrm{MPa}$. For the fluid, a perfect gas is assumed with the inlet pressure set to $0.2026 \mathrm{MPa}$, and the outlet pressure to $0.1013 \mathrm{MPa}$.

Transient numerical solutions are obtained with matching and non-matching meshes for this flexible inhibitor problem. Snapshots of the fluid region in the vicinity of the deflected inhibitor are shown in Figure 18 for the common-refinement based and node-projection schemes. As expected, the pressure difference building across the inhibitor leads to its substantial bending.

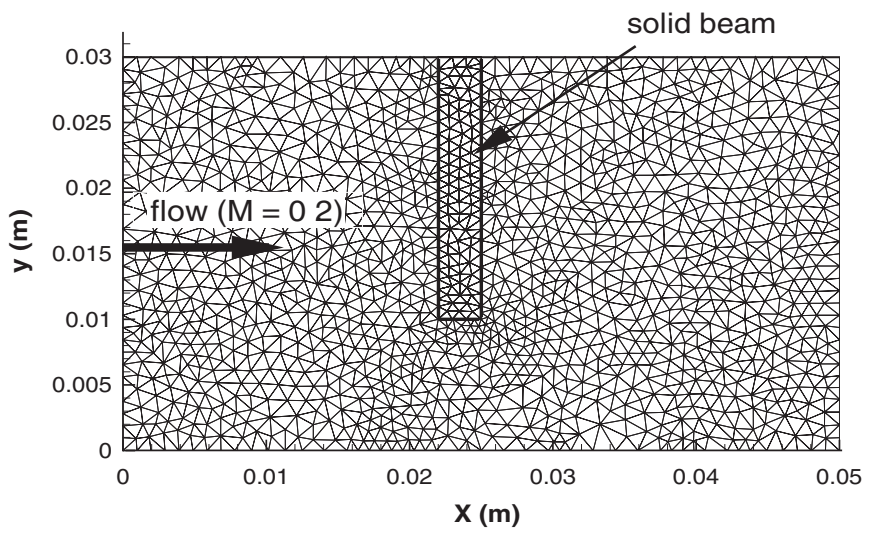

Figure 17. Geometry and discretization of beam bending problem. 

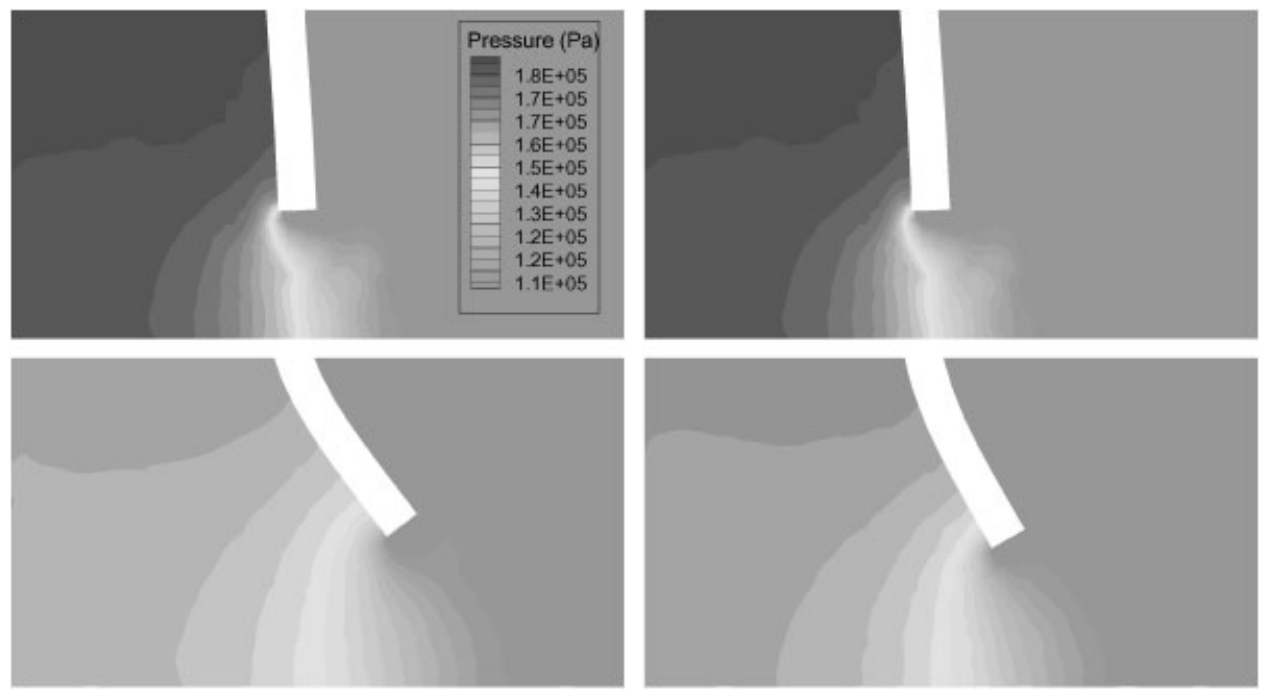

Figure 18. Pressure contour of beam bending problem at $1 \mathrm{~ms}$ (top) and $3 \mathrm{~ms}$ (bottom). The left plots correspond to the common-refinement based scheme and the right plots to the node-projection scheme.

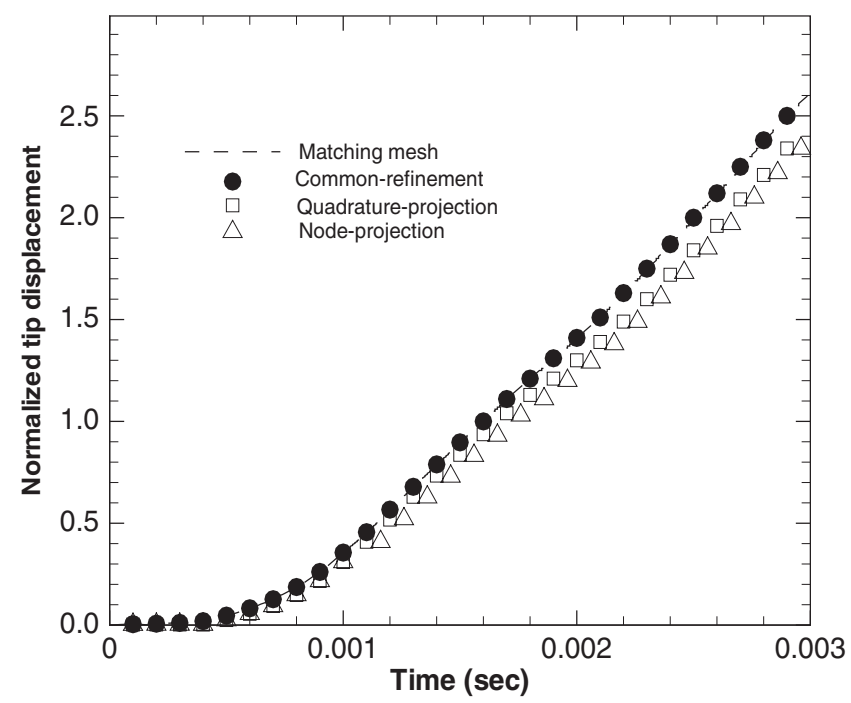

Figure 19. Comparison of beam tip deflection for three load transfer schemes with reference matching solution.

As shown in Figure 18, the solutions obtained with the common-refinement and nodeprojection schemes are very similar initially. However, at later times, the solution of the nodeprojection scheme seems to yield a smaller deflection of the inhibitor, which also affects 
Table II. Computational statistics of three fluid-solid interaction problems.

\begin{tabular}{|c|c|c|c|c|c|}
\hline \multirow[b]{2}{*}{ Test case } & \multicolumn{3}{|c|}{ Interface computation (s) } & \multicolumn{2}{|c|}{ Solvers (s) } \\
\hline & NP & QP & $\mathrm{CR}$ & ALE fluid & Solid \\
\hline Elastic piston & 0.435 & 0.452 & 0.467 & 47.12 & 2.31 \\
\hline Superseismic shock & 0.899 & 0.968 & 0.972 & 51.28 & 3.38 \\
\hline Beam bending & 1.832 & 1.941 & 1.956 & 74.72 & 5.91 \\
\hline
\end{tabular}

the flow solution. The increasing difference between the solutions provided by the two load transfer schemes is also illustrated in Figure 19, which presents the evolution of the magnitude of the tip displacement vector (normalized by the initial thickness of the beam) for the first $3 \mathrm{~ms}$ as obtained from the three load transfer schemes (symbols) and the matching mesh case (dashed curve). Similar to the previous results, the common-refinement based scheme yields a tip displacement evolution effectively identical to that obtained in the matching reference solution. However, the traditional schemes yield tip displacements that are consistently lower, with an error of about $8 \%$ at $3.0 \mathrm{~ms}$.

\section{COST EVALUATION AND 3-D ASPECTS}

Computational costs are an important factor when selecting a numerical scheme for largescale simulations. In the context of general aeroelastic applications, the size of the interface discretization is typically orders of magnitude smaller than the fluid and solid meshes. Therefore, the cost of interface computation typically represents a small fraction of the overall cost, which is quantified in the following.

In Reference [6], the asymptotic analysis of computation and storage costs of various data transfer scheme have been summarized. To assess the cost associated with the load transfer, we report hereafter those obtained on the FSI problems studied earlier. In this assessment study, we have computed the cost as time spent during converting the fluid tractions to the load vectors on solid side. Note that it is not necessary to solve a linear system to convert the load vectors to the tractions on the solid interface nodes in the quadrature-projection and common-refinement based schemes, as the load vectors are used directly by the finite element solver of the solid side.

The computational statistics of each fluid-solid interaction problem are tabulated for the three load transfer schemes in Table II, where NP, QP and CR indicates node-projection, quadratureprojection, and common-refinement. All the computations are performed on a $750 \mathrm{MHz}$ UltraSPARC III CPU Sun Server with a 8.0 GByte of main memory. We ran each case for 1000 iterations using the same time step for the fluid, mesh motion and solid solvers. As apparent in Table II, the interface computation time increases as the number of interface nodes increases for the three problems, but the computation time is less than $2 \%$ of the overall CPU time in all cases. The common-refinement based scheme is only slightly more expensive than the other two load transfer schemes, but this increase is negligible, compared to the overall costs. 
All three of the load transfer schemes can be extended to 3-D, however the commonrefinement based scheme introduces the additional difficulty of creating a common refinement surface. Such an algorithm for computing the common refinement has been recently implemented in 3-D [14]. In this approach, the common refinement of two surface meshes is defined by a common surface of arbitrary shape by overlaying them on top of each other for the intersection of elements. As far as the computational costs for 3-D problems are concerned, the interface computations for the surface meshes are insignificant as compared to the volumetric meshes for the fluid and the solid solvers [19].

\section{CONCLUSION}

We have systematically assessed three different conservative load transfer schemes, namely, node-projection, quadrature-projection, and common-refinement based schemes for non-matching meshes. In the traditional node- and quadrature-projection schemes, the integrand exhibits discontinuities that generally lead to load transfer errors depending on the fluid-solid mesh mismatch along the interface. These errors correlate well to a grid mismatch function developed in this study and they not only affect the solution of the interface, but also can significantly undermine the solutions in the fluid and solid subdomains. By utilizing a common refinement and integrating over the subintervals, the error becomes independent of the mismatching along the interface, such that solution with non-matching meshes was effectively identical to the solution of the matching meshes. These findings were demonstrated first with the aid of a simple 1-D static load transfer analysis, then through two classical transient fluid-solid interaction problems and finally, with the aid of a flexible beam bending problem involving large deformations. The common-refinement based scheme was found to be have a cost similar to that of the traditional load transfer schemes. Another interesting subject of non-matching interfaces is to adapt the interface meshes to minimize errors, which we are exploring using the common-refinement based scheme.

\section{APPENDIX A: ANALYTICAL SOLUTION OF SUPERSEISMIC SHOCK PROBLEM}

As shown in Figure 14, the superseismic shock problem consists of an isotropic, linearly elastic solid subjected to shock traveling at superseismic speed $V_{\mathrm{sh}}$ in the adjacent fluid domain. Let $c_{\mathrm{d}}=\sqrt{(E(1-v)) /\left(\rho_{\mathrm{s}}(1+v)(1-2 v)\right)}$ and $c_{\mathrm{S}}=\sqrt{E /\left(2 \rho_{\mathrm{s}}(1+v)\right)}$, respectively, denote the dilatational and shear wave speeds under plain strain conditions.

Due to the superseismic velocity of the shock, Mach cones corresponding to the p- and s-waves appear in the solid domain with respective inclinations $\alpha_{p}$ and $\alpha_{s}$ are given by Kolsky [20]

$$
\tan \left(\pi / 2-\alpha_{\mathrm{p}}\right)=\sqrt{V_{\mathrm{sh}}^{2} / c_{\mathrm{d}}^{2}-1}, \quad \tan \left(\pi / 2-\alpha_{\mathrm{s}}\right)=\sqrt{V_{\mathrm{sh}}^{2} / c_{\mathrm{s}}^{2}-1}
$$

Let $\mathbf{n}_{\delta}$ denote the normal to the outer interface surface deformed by the pressure jump $\Delta p$ behind the shock. The interface velocities in the $x$ - and $y$-directions are related to the applied 
pressure jump, the shock speed and the material properties, and they can be expressed as

$$
\left[\begin{array}{c}
\dot{u}_{x, I} \\
\dot{u}_{y, I}
\end{array}\right]=\frac{\Delta p V_{\mathrm{sh}}(1-v)}{2 \rho_{\mathrm{s}} c_{\mathrm{d}}^{2} N}\left[\begin{array}{c}
\cos 2 \alpha_{\mathrm{s}}-\cos 2\left(\alpha_{\mathrm{p}}-\alpha_{\mathrm{s}}\right) \\
\sin 2 \alpha_{\mathrm{p}}
\end{array}\right]
$$

where $N=\cos ^{2} \alpha_{\mathrm{s}}+(1-2 v) \cos ^{2}\left(\alpha_{\mathrm{s}}-\alpha_{\mathrm{p}}\right)-1+v$. The deflection angle $\delta$ is then given by

$$
\tan \delta=\frac{\dot{u}_{y, I}}{V_{\mathrm{sh}}+\dot{u}_{x, I}}
$$

In the fluid domain, let us denote the pressure, density and speed of sound in the undisturbed region by $p_{1}, \rho_{1}$ and $c_{1}=\sqrt{\left(\gamma p_{1} / \rho_{1}\right)}$, respectively. Behind the shock, these quantities become $p_{2}=p_{1}+\Delta p, \rho_{2}$ and $c_{2}=\sqrt{\left(\gamma p_{2} / \rho_{2}\right)}$. The Mach number of the undisturbed fluid particle is

$$
M_{1}=\frac{V_{\mathrm{sh}}}{c_{1}}=\left[\frac{6 p_{2} / p_{1}+1}{7}\right]^{1 / 2}
$$

and the shock angle $\beta$ can be calculated from the deflection $\delta$ of the flow using plane oblique shock theory as

$$
\tan \delta=\frac{2 \cot \beta\left(M_{1}^{2} \sin ^{2} \beta-1\right)}{\left((\gamma+1) M_{1}^{2}-2\left(M_{1}^{2} \sin ^{2}(\beta)-1\right)\right)}
$$

Further, the pressure jump $\Delta p$ can be expressed as

$$
\frac{\Delta p}{p_{1}}=\frac{2 \gamma}{\gamma+1}\left(M_{1}^{2} \sin ^{2} \beta-1\right)
$$

Equations (A1)-(A6) yield a system of coupled non-linear equations for $\delta, \beta$ and $\Delta p$.

\section{ACKNOWLEDGEMENTS}

The authors wish to acknowledge support from the Center for Simulation of Advanced Rockets (CSAR) [funded by the U.S. Department of Energy] under subcontract B523819 and from the Computational Science and Engineering (CSE) program at the University of Illinois. The authors thank anonymous referees for their helpful comments in improving the presentation of this paper.

\section{REFERENCES}

1. Ghattas O, Li X. A variational finite element method for stationary nonlinear fluid-solid interaction. Journal of Computational Physics 1995; 121:347-356.

2. Piperno S, Farhat C, Larrouturou B. Partitioned procedures for the transient solution of coupled aeroelastic problems-part II: energy transfer analysis and three-dimensional applications. Computer Methods in Applied Mechanics and Engineering 2001; 190:3147-3170.

3. Piperno S, Farhat C. Partitioned procedures for the transient solution of coupled aeroelastic problems. Part I: Model problem, theory, and two-dimensional application. Computer Methods in Applied Mechanics and Engineering 1995; 124:79-112.

4. Farhat C, Lesoinne M, LeTallec P. Load and motion transfer algorithms for fluid/structure interaction problems with non-matching discrete interfaces: momentum and energy conservation, optimal discretization and application to aeroelasticity. Computer Methods in Applied Mechanics and Engineering 1998; 157: $95-114$. 
5. Cebral JR, Löhner R. Conservative load projection and tracking for fluid-structure problems. AIAA Journal 1997; 35:687-692.

6. Jiao X, Heath MT. Common-refinement based data transfer between nonmatching meshes in multiphysics simulations. International Journal for Numerical Methods in Engineering 2004; 61:2402-2427.

7. Farhat C, Lesoinne M, Maman N. Mixed explicit/implicit time integration of coupled aeroelastic problems: three field formulation, geometric conservation and distributed solution. International Journal for Numerical Methods in Engineering 1995; 21:807-835.

8. Smith MJ, Hodges DH, Cesnik CES. Evaluation of computational algorithms suitable for fluid-structure interactions. Journal of Aircraft 2000; 37:282-294.

9. Michler C, van Brummelen EH, Hulshoff SJ, de Borst R. The relevance of conservation for stability and accuracy of numerical methods for fluid-structure interaction. Computer Methods in Applied Mechanics and Engineering 2003; 192:4195-4215.

10. Cai XC, Dryja M, Sarkis M. Overlapping nonmatching grid mortar element methods for elliptic problems. SIAM Journal on Numerical Analysis 1999; 36:581-606.

11. Simo JC, Wriggers P, Taylor RL. A perturbed Lagrangian formulation for the finite element solution of contact problems. Computer Methods in Applied Mechanics and Engineering 1985; 50:163-180.

12. Cebral JR. Loose-coupling algorithms for fluid-structure interaction. Ph.D. Thesis, George Mason University, 1996.

13. Ramshaw JD. Conservative rezoning algorithm for generalized two-dimensional meshes. Journal of Computational Physics 1985; 59:193-199.

14. Jiao X, Heath MT. Overlaying surface meshes (part 1): algorithms. International Journal of Computational Geometry and Applications 2004; 14:379-402.

15. Bleich HH, Matthews A, Wright JP. Step load moving with superseismic velocity on the surface of a half-space of granular material. Technical Report AFWL-TR-65-69, 1965.

16. Arienti M, Hung P, Morano E, Shepherd E. A level set approach to Eulerian-Lagrangian coupling. Journal of Computational Physics 2003; 185:213-251.

17. Fiedler RA, Breitenfeld MS, Jiao X, Geubelle PH, Guoy D, Brandyberry M. Simulations of slumping propellant and flexible inhibitor in solid rocket motors. AIAA 2002-4341 CP, 2002.

18. Weaver MA, Gramoll KC, Roach RL. Structural analysis of a flexible structural member protruding into an interior flow field. AIAA 93-1446 CP, 1993.

19. Heath MT, Jiao X. Parallel simulation of multicomponent systems. High Performance Computing for Computational Science-VECDAR/2004 (LNCS 3402), Valencia, Spain, June 2004; 496-513.

20. Kolsky H. Stress Waves in Solids. Dover: New York, 1963.

21. Heath MT. Scientific Computing: An Introduction Survey (2nd edn). McGraw-Hill: New York, 2002. 